\title{
Banner Evaluation Predicted by Eye Tracking Performance and the Median Thinking Style
}

\author{
Man-Ying Wang ${ }^{1}$, Da-Lung Tang ${ }^{2}$, Chih-Tung Kao ${ }^{1}$, and Vincent C. Sun ${ }^{3}$ \\ ${ }^{1}$ Department of Psychology, Soochow University, Taipei, Taiwan \\ mywang@scu.edu.tw, \{athena1011, daluntang\}@gmail.com \\ ${ }^{2}$ Department of Mass Communication, Tam Kang University, Taipei, Taiwan \\ ${ }^{3}$ Department of Mass Communication, Chinese Culture University, Taipei, Taiwan \\ csunefaculty.pccu.edu.tw
}

\begin{abstract}
The current study examined whether and how the Chinese culture rooted median thinking style may affect banner ad viewing and evaluation. Eye tracking performance was recorded as participants viewed banner ads of different information complexity. High median thinking participants were characterized by a flexible perceptual processing style. Their eye tracking performance showed that they responded to information complexity of the banner ads and attempted to integrate information spatially for low complexity banners. Less effortful (and more fluent) eye tracking performance was associated with more positive banner evaluation and the relationship was mediated by experienced fluency in high median thinkers. Information complexity also guided eye tracking. These findings demonstrated the potential of eye tracking measures in predicting effects of culture (and design) related factors on banner evaluation.
\end{abstract}

Keywords: banner advertisements, eye tracking, median thinking, ZhongYong, information complexity.

\section{Introduction}

It is well known that banner ads are typically ignored by viewers of a webpage (i.e., "banner blindness" as dubbed by[1])[2], [3]. However, other studies showed evidence of banner viewing or that some very general information could be captured by the viewer in a brief glance of the ad [4], [5]. Task goal of the user could be an important moderator of how attention is directed towards specific banner ad and search behavior in general [6], [7]. As a result, it is probably less fruitful to search for an optimal banner design than to understand the effect of user characteristics on the design of banner ads [7], [8]. An important aspect of user characteristic that has received less attention is on how culturally related individual differences in visual information processing may affect viewer's intention to click on the ad, given the brief processing time or limited processing resources allocated to the banner ad.

A culturally cultivated thinking style - median thinking (also known as ZhongYong, doctrine of the mean or midway thinking) (see [9], for a review) was the focus of this study. Median thinking is historically rooted in the Chinese philosophy of life that guides the deliberation, selection, and execution of action in daily life. It emphasized 
that one should "....consider things carefully from different perspectives, avoid going to extremes, behave in situationally appropriate ways, and maintain interpersonal harmony...." [10] (p 158).

Median thinking entails concrete cognitive and perceptual strategies to support the fulfillment of the above interpersonal goals. The wait-and-see cognitive processing style is conducive to the thorough examination of the situation before making any actions as well as the holding back of extreme emotions [9]. In a study using the Posner cuing paradigm, participants of high median thinking were less affected by the emotionality of cues than low median thinking participants regardless of the validity of the cue [11]. These findings suggested that high-median thinking participants tend to withhold the processing of emotional information and consider the nature of the situation first. High median thinking is also characterized by a global-yet-flexible perceptual style. Relative to low median thinkers, they preferred global to local processing and showed higher flexibility in the switch between global and local processing modes, especially at the presence of emotional primes [12]. As such, the viewer could quickly derive the gist of the visual scene (from global processing) based on which to determine the need to proceed with local processing or to remain at the global level of analysis.

The current study was focused on the global-yet-flexible processing style of median thinkers and its relationships with banner ad complexity and evaluation. Previous studies of visual scene processing demonstrated an increase in fixation duration and a decrease in saccade amplitude as viewing proceeded. They were also considered to be the hallmarks of global-to-local processing [13], [14]. Shorter fixations and larger saccades dominate during earlier global processing (first $2 \mathrm{~s}$.) when the gist of a scene is captured. Object-centered analysis or local processing is performed later that is characterized by longer fixations and smaller saccades [14]. Since the gist of a banner ads is quickly available to the viewer (i.e., in the first $100 \mathrm{~ms}$ ) [5], it is expected that visual behavior during early viewing of a banner could predict the viewer's evaluation or the intention to click through the ad. To reveal the relationship between viewing behavior and banner ad attitude, eye tracking performance of the viewer was recorded as they viewed each banner ad after which they gave evaluative responses towards whether the banner ad appeared clear and simple, was rich in information and whether the viewer would like to click on it.

High median thinking viewers who exhibited a global-yet-flexible perceptual style were expected to recognize the gist of a banner using global processing and switch to the detailed or local processing of product information more efficiently than low median thinkers. Their global/local processing was also expected to be more responsive than low median thinkers to information complexity manipulation since global and local processors are likely to also accommodate with the variation in complexity. For example, global processing could be sufficient to deal with needs to comprehend low complexity banner ads while local processing predominates in a high complexity ad in order to process the large number of details. Specifically, faster global-to-local switch (characterized by the increase in fixation duration and the decrease in saccade amplitude into viewing) [13], [14], was expected for high median thinking participants. Information complexity measured by the entropy measure in information theory [15] was expected to also affect the efficiency of global-local switch so that the switch was faster as complexity increased, especially for high median thinking participants. 


\section{$2 \quad$ Method}

\subsection{Participants}

Participants were forty-nine female undergraduate students who were compensated NT\$ 100 for their time.

\subsection{Design and Materials}

The design was a median thinking (2) x banner complexity (3) mixed design with median thinking serving as a between-subject factor and banner complexity being within-subjects.

Participants filled out the ZhongYong Belief-Value Scale [16] to evaluate their propensity to median thinking. Each banner ad subtended approximately $31^{\circ}$ in width and $14^{\circ}$ in height and the fixation cross subtended approximately $0.8^{\circ} \times 0.8^{\circ}$ on the computer screen. Twenty-four banner ads of female apparels were collected from the internet that were divided into three levels of information complexity according to the computed information entropy measure (see [17] for detailed descriptions of information entropy calculation). Using seven-point scales, three evaluative responses were probed for each banner, i.e., "The layout in the ad is clear", "Product information in the banner ad is rich", "I would like to click on the ad".

\subsection{Procedure}

The experiment was conducted in a sound attenuated, softly lighted room. Participants were seated $60 \mathrm{~cm}$ in front of an HP-L1706 monitor. The experiment was controlled by a Visual Basic program run on a Core2 Duo E7400 $(2.8 \mathrm{GHz})$ personal computer. Eye movements were recorded by the SR Research EyeLink 1000 System at the sampling rate of $500 \mathrm{~Hz}$. The tracking was monocular and conducted in the remote mode. Fixations, saccades and eye blinks were determined using the default definition of the EyeLink System. A nine-point calibration procedure was conducted and the calibration was validated and repeated when necessary until the optimal calibration criterion was met.

Participants were asked to evaluate a series of ads when their eye movements were recorded. The experiment stared after 4 practice trials (repeated if necessary) and the calibration procedure. Each trial began with a fixation cross presented at the center of the screen. Participants moved the mouse cursor to the center location of the cross in order to start the presentation of the banner ad. Each banner was presented for five seconds followed by the three rating questions. The question was presented on the top and the seven-point rating scale was presented at the bottom. Participants indicated their responses by moving the cursor to the location of the corresponding digit. They were given 9 seconds to respond to each question.

\section{Results}

Fixation durations that were less than $90 \mathrm{~ms}$ or greater than $1500 \mathrm{~ms}$ were excluded from the analysis. Participants who scored at the top one third on their ZhongYong 
scores were treated as high in median thinking and those at the bottom one third were treated as low in median thinking (the cutoffs were 36 and 43 respectively).

\subsection{Banner Ratings}

Each of the three banner ratings were submitted to median thinking (2) x information complexity ANOVAs. For all three ratings, information complexity was the only significant effect (Clarity: $F(2,66)=13.24, p<.0001$; Richness: $F(2,66)=65.70, p<$ .0001 ; Clicking: $F(2,66)=9.76, p<.0005)$. Clarity rating: low $>$ high, medium $>$ high (both $p s<.0005)$, medium $=$ low. Richness rating: high $>$ medium $>$ low (all $p \mathrm{~s}<$ $.0001)$. Willingness to click: medium $>$ low $(p<.005)$, high $>$ low $(p<.001)$, medium $=$ high $($ see Table 1$)$.

Table 1. The effect of information complexity on ratings of clarity, information richness and the willingness to click. Means and standard deviations are listed.

\begin{tabular}{crrrrrr}
\hline Information complexity & \multicolumn{2}{c}{ Clarity } & \multicolumn{2}{c}{ Richness } & \multicolumn{2}{c}{ Clicking } \\
\hline low & 4.93 & .87 & 3.27 & .78 & 3.85 & .91 \\
medium & 4.90 & .65 & 4.17 & .68 & 4.44 & .59 \\
high & 4.39 & .80 & 4.83 & .83 & 4.47 & 1.08 \\
\hline
\end{tabular}

\subsection{Eye Tracking Parameters}

Mean fixation duration, mean saccade amplitude, mean fixation number, total fixation duration and total scan path were separately submitted to median thinking (2) $\mathrm{x}$ information complexity (3) ANOVA. The only significant effect involving median thinking was the interaction between median thinking and complexity for total scan path $(F(2,66)=3.68, p<.05)$ (see Table 2 ). Total scan path was larger in high than low median thinking participants for low complexity banners $(p<.0005)$ but the difference was not significant for medium or high complexity banners $(p s=.68, .75)$. For all eye tracking measures analyzed, the effect of information complexity was significant (see Table 3): mean fixation duration $(F(2,66)=73.92, p<.0001)$, medium $>$ low $>$ high; mean saccade amplitude $(F(2,66)=18.13, p<.0001)$, low $>$ medium $=$ high; number of fixations $(F(2,66)=90.73, p<.0001)$, high $>$ low $>$ medium; total fixation duration $(F(2,66)=5.81, p<.005)$, high=medium $>$ low; total scan path $(F(2,66)=49.37, p<.0001)$, low $=$ high $>$ medium.

Table 2. The effect of median thinking and information complexity on the total scan path (in degrees of visual angle). Means and standard deviations are listed.

\begin{tabular}{ccccccc}
\hline & \multicolumn{6}{c}{ Information complexity } \\
\cline { 2 - 7 } Median thinking & \multicolumn{5}{c}{ low } & \multicolumn{3}{c}{ medium } & \multicolumn{3}{c}{ high } \\
\hline low & 77.3 & 11.5 & 70.6 & 12.8 & 83.0 & 14.8 \\
high & 86.1 & 13.1 & 73.3 & 12.7 & 85.6 & 14.0 \\
\hline
\end{tabular}


Table 3. The effect of informaiton complexity on eye tracking parameters. Means and standard deviations are listed.

\begin{tabular}{crrrrrr}
\hline & \multicolumn{6}{c}{ Information complexity } \\
\cline { 2 - 7 } Eye tracking parameters & \multicolumn{2}{c}{ low } & \multicolumn{3}{c}{ medium } & \multicolumn{2}{c}{ high } \\
\hline mean fixation duration & 252.0 & 32.5 & 262.4 & 29.8 & 228.1 & 24.2 \\
mean saccade amplitude & 4.77 & .56 & 4.34 & .63 & 4.45 & .53 \\
number of fixations & 16.9 & 1.7 & 16.5 & 1.5 & 18.7 & 1.6 \\
total fixation duarion & 4071.2 & 186.8 & 4150.7 & 190.6 & 4112.0 & 172.7 \\
total scan path & 82.1 & 13.0 & 72.0 & 12.6 & 84.4 & 14.2 \\
\hline
\end{tabular}

Table 4. The quadratic model fit for fixation duration as the function of banner complexity, median thinking and the viewing period

\begin{tabular}{|c|c|c|c|c|c|c|c|c|}
\hline \multirow[t]{2}{*}{ Condition } & \multirow[t]{2}{*}{ time } & \multicolumn{2}{|l|}{ Linear } & \multicolumn{2}{|l|}{ Quadratic } & \multicolumn{2}{|l|}{ Intercept } & \multirow[t]{2}{*}{$\mathrm{R}^{2}$} \\
\hline & & Est & SE & Est & SE & Est & SE & \\
\hline low complexity & & & & & & & & \\
\hline \multirow[t]{3}{*}{ low median thinking } & $1 \mathrm{~s}$ & 152.4 & 153.2 & -86.9 & 125.3 & $172.7^{*}$ & 40.2 & .53 \\
\hline & $2 \mathrm{~s}$ & 86.1 & 41.4 & -22.4 & 18.3 & $185.6^{* * *}$ & 19.8 & .61 \\
\hline & $5 s$ & $45.9 * * *$ & 10.9 & $-7.8 * *$ & 2.2 & $204.0 * * *$ & 11.3 & .71 \\
\hline \multirow[t]{3}{*}{ high median thinking } & $1 \mathrm{~s}$ & $349.7^{*}$ & 64.8 & $-224.4^{*}$ & 52.9 & $109.5^{*}$ & 17.0 & .97 \\
\hline & $2 \mathrm{~s}$ & $152.2 * *$ & 28.9 & $-54.2 * *$ & 12.8 & $153.5 * * *$ & 13.8 & .86 \\
\hline & $5 s$ & $45.3 * * *$ & 10.9 & $-7.5 * *$ & 2.2 & $197.0^{* * *}$ & 11.3 & .55 \\
\hline \multicolumn{9}{|l|}{ medium complexity } \\
\hline \multirow[t]{3}{*}{ low median thinking } & $1 \mathrm{~s}$ & 197.4 & 96.5 & -105.0 & 78.9 & $175^{*}$ & 25.3 & .89 \\
\hline & $2 \mathrm{~s}$ & $128.8^{*}$ & 40.8 & $-46.9^{*}$ & 18.5 & $190.0 * * *$ & 20.0 & .66 \\
\hline & $5 s$ & $38.4 * *$ & 12.9 & $-6.8^{*}$ & 2.6 & $226.8 * * *$ & 13.5 & .34 \\
\hline \multirow{3}{*}{ high median thinking } & $1 \mathrm{~s}$ & 238.3 & 75.0 & -89.0 & 61.3 & $130.5^{*}$ & 19.7 & .98 \\
\hline & $2 s$ & $192.9 * * *$ & 23.9 & $-64.0 * * *$ & 10.6 & $144.2 * * *$ & 11.4 & .95 \\
\hline & $5 s$ & $50.8^{* *}$ & 16.4 & $-8.6^{*}$ & 3.3 & $209.7 * * *$ & 17.1 & .38 \\
\hline \multirow{4}{*}{$\begin{array}{l}\text { high complexity } \\
\text { low median thinking }\end{array}$} & & & & & & & & \\
\hline & $1 \mathrm{~s}$ & 147.7 & 60.0 & -94.4 & 49.0 & $166.0 * *$ & 15.8 & .86 \\
\hline & $2 \mathrm{~s}$ & 32.4 & 21.7 & -4.2 & 9.6 & $194.2 * * *$ & 10.4 & .76 \\
\hline & $5 s$ & $22.9 * *$ & 6.9 & $-3.2^{*}$ & 1.4 & $201.2 * * *$ & 7.2 & .55 \\
\hline \multirow[t]{3}{*}{ high median thinking } & $1 \mathrm{~s}$ & 97.5 & 31.6 & -47.7 & 25.9 & $178.9 * *$ & 8.3 & .96 \\
\hline & $2 \mathrm{~s}$ & $75.5^{* *}$ & 20.5 & -26.4 & 9.1 & $182.9^{* * *}$ & 9.8 & .76 \\
\hline & $5 \mathrm{~s}$ & $27.6^{* *}$ & 7.4 & $-4.9 * * *$ & * 1.5 & $201.7 * * *$ & 7.8 & .44 \\
\hline
\end{tabular}

A quadratic regression model was used to fit the mean fixation duration on time of viewing. Table 4 listed all fitted parameters for time periods of 1, 2, and 5 seconds. The parameter for the linear component indicated the speed of increase in fixation duration over viewing time. For the $5 \mathrm{~s}$. fit, this parameter was positive and significant for high and low median thinker at all complexity levels. However, for high median thinking participants, the linear parameter was significant at all of the time period examined when banner complexity was low, significant at $2 \mathrm{~s}$ and $5 \mathrm{~s}$ when banner 




Fig. 1. The scatter plot of fixation duration as the function of information complexity and median thinking overlaid with the fitted quadratic function

I

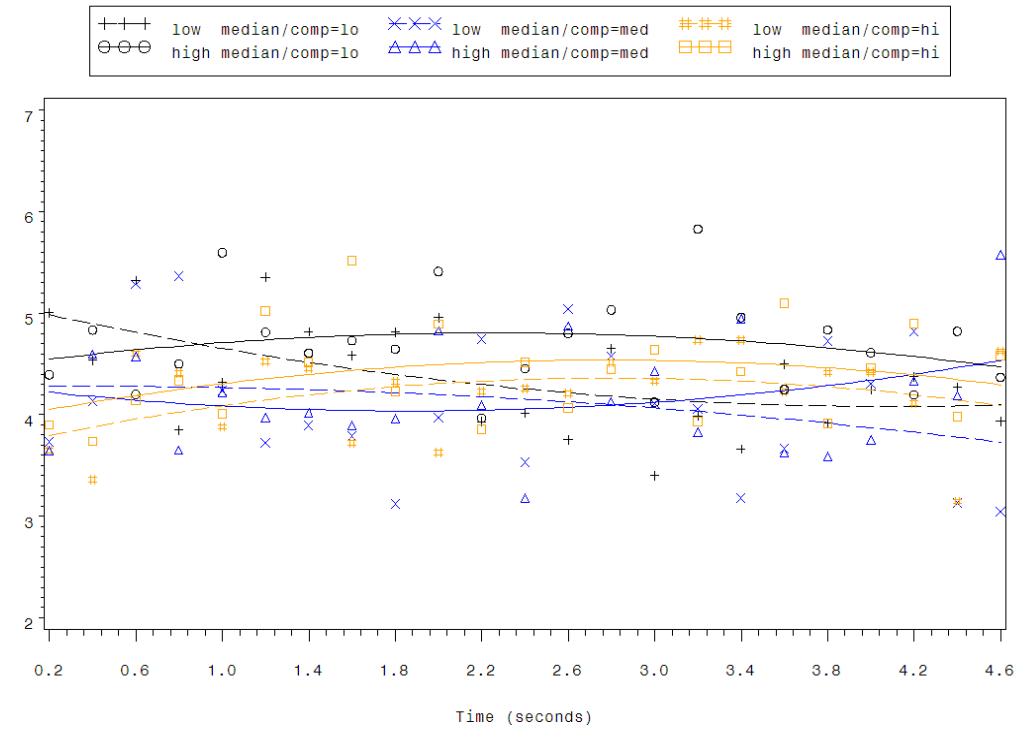

Fig. 2. The scatter plot of saccade amplitude as the function of information complexity and median thinking overlaid with the fitted quadratic function 
complexity was medium and significant at $5 \mathrm{~s}$ of viewing period when banner complexity was high (see Table 4 and Fig. 1). For high-median thinking participants, the global-to-local switch appeared to be earlier the lower the complexity of the banner ad was. The lack of similar pattern for low median thinking participants suggested that high median thinkers were more sensitive and responded to banner complexity by modifying the time of global-to-local switch.

As seen in Fig 2, there apparently lacks any clear quadratic trend or linear trend for saccade amplitude data. None of the linear (or quadratic) parameters was significant regardless of the level of information complexity and median thinking (see Table 5).

Table 5. The quadratic model fit for saccade amplitude as the function of banner complexity, median thinking and the viewing period

\begin{tabular}{|c|c|c|c|c|c|c|c|c|}
\hline \multirow[t]{2}{*}{ Condition } & \multirow[t]{2}{*}{ time } & \multicolumn{2}{|l|}{ Linear } & \multicolumn{2}{|c|}{ Quadratic } & \multicolumn{2}{|c|}{ Intercept } & \multirow[t]{2}{*}{$\mathrm{R}^{2}$} \\
\hline & & Est & SE & Est & SE & Est & SE & \\
\hline \multicolumn{9}{|l|}{ low complexity } \\
\hline \multirow[t]{3}{*}{ low median thinking } & $1 \mathrm{~s}$ & -.19 & 5.48 & -.70 & 4.48 & 5.03 & 1.44 & .33 \\
\hline & $2 \mathrm{~s}$ & -.75 & 1.23 & .36 & .55 & $5.02 * * *$ & .59 & .06 \\
\hline & $5 s$ & -.49 & .30 & .06 & .06 & $5.08 * * *$ & .32 & .30 \\
\hline \multirow[t]{3}{*}{ high median thinking } & $1 \mathrm{~s}$ & -3.77 & 3.72 & 4.01 & 3.04 & $5.21 *$ & .98 & .66 \\
\hline & $2 \mathrm{~s}$ & .32 & 1.09 & -.01 & .48 & $4.43 * * *$ & .52 & .18 \\
\hline & $5 s$ & .28 & .31 & -.06 & .06 & $4.49 * * *$ & .32 & .05 \\
\hline \multicolumn{9}{|l|}{ medium complexity } \\
\hline \multirow[t]{3}{*}{ low median thinking } & $1 \mathrm{~s}$ & 9.95 & 3.76 & -7.35 & 3.07 & 1.82 & .99 & .80 \\
\hline & $2 \mathrm{~s}$ & 1.13 & 1.61 & -.75 & .71 & $4.04 * *$ & .77 & .32 \\
\hline & $5 s$ & .02 & .45 & -.03 & .09 & $4.28 * * *$ & .47 & .07 \\
\hline \multirow[t]{3}{*}{ high median thinking } & $1 \mathrm{~s}$ & 3.62 & 4.78 & -2.94 & 3.91 & 3.26 & 1.25 & .22 \\
\hline & $2 s$ & -.66 & 1.08 & .35 & .48 & $4.33 * * *$ & .51 & .10 \\
\hline & $5 s$ & -.25 & .36 & .07 & .07 & $4.27 * * *$ & .38 & .07 \\
\hline \multicolumn{9}{|l|}{ high complexity } \\
\hline \multirow[t]{3}{*}{ low median thinking } & $1 \mathrm{~s}$ & 4.89 & 4.41 & -3.44 & 3.60 & 2.56 & 1.16 & .46 \\
\hline & $2 \mathrm{~s}$ & 2.08 & .99 & -.90 & .44 & $3.16^{* * *}$ & .48 & .39 \\
\hline & $5 s$ & .47 & .28 & -.08 & .06 & $3.70 * * *$ & .29 & .14 \\
\hline \multirow[t]{3}{*}{ high median thinking } & $1 \mathrm{~s}$ & 1.58 & 2.02 & -.98 & 1.25 & $3.51 *$ & .53 & .42 \\
\hline & $2 \mathrm{~s}$ & 1.39 & 1.11 & -.35 & .49 & $3.43 * * *$ & .53 & .50 \\
\hline & $5 s$ & .41 & .30 & -.06 & .06 & $3.97 * * *$ & .32 & .09 \\
\hline
\end{tabular}

\subsection{Correlation between Eye Tracking Measures and Banner Evaluation}

The correlation pattern in Table 6 (based on data from all participants) showed that the total scan path correlated negatively with participants' ratings of how willing they were to click on the ad. Willingness to click also correlated positively with mean fixation duration, negatively with the mean number of fixation and mean saccade amplitude. In other words, shorter total scan path and mean saccade amplitude, small-er number of fixations as well as longer mean fixation durations were consistent with higher willingness ratings of banner ad clicking. 
A mediation analysis was performed to examine whether the relationship between the total scan path and the willingness to click was mediated by processing fluency [18]. Data from all participants were used in the analysis. The Sobel tests were separately performed on high and low median thinking participants (based on a median split) using clarity rating as the measure of processing fluency. For high median thinkers, processing fluency mediated the relationship between total scan path and willingness to click no matter the analysis was based on one or five seconds of viewing behavior ( $\mathrm{ps}=.003, .004)$. For low median thinking participants, however, processing fluency did not mediate the relationship.

Table 6. Correlations among eye tracking measures and banner evaluative responses $\left({ }^{*} p<.05\right.$, $* * p<.01, * * * p<.001)$

\begin{tabular}{|c|c|c|c|c|c|c|c|c|c|}
\hline & Clarity & Richness & licking & $\begin{array}{l}\text { mean } \\
\text { fix. dur. }\end{array}$ & $\begin{array}{l}\text { mean } \\
\text { sacc. } \\
\text { amp. }\end{array}$ & $\begin{array}{l}\text { no. of } \\
\text { fix. }\end{array}$ & $\begin{array}{c}\text { total } \\
\text { fix. dur. }\end{array}$ & $\begin{array}{l}\text { total } \\
\text { scan } \\
\text { path }\end{array}$ & $\begin{array}{c}\text { Zhong } \\
\text { Yong }\end{array}$ \\
\hline \multicolumn{10}{|l|}{ Clarity } \\
\hline Richness & $.48 * * *$ & 1.00 & & & & & & & \\
\hline Clicking & $.44 * *$ & $.69 * * *$ & 1.00 & & & & & & \\
\hline mean fix. dur. & .27 & .24 & $.29 *$ & 1.00 & & & & & \\
\hline mean sacc. amp. & .22 & $-.34 *$ & -.23 & -.27 & 1.00 & & & & \\
\hline no. of fix. & -.25 & -.24 & -.27 & $-.93 * * *$ & $.34 * *$ & 1.00 & & & \\
\hline total fix. dur. & .01 & .10 & .14 & $.67 * * *$ & -.27 & $-.45 * *$ & 1.00 & & \\
\hline total scan path & -.27 & $-.39 * *$ & $-.30^{*}$ & $-.67 * * *$ & $.81 * * *$ & $.66^{* * *}$ & $-.43 * *$ & 1.00 & \\
\hline ZhongYong & -.05 & -.03 & .02 & .06 & .09 & .03 & .18 & .08 & 1.00 \\
\hline
\end{tabular}

\section{Discussion}

The current study examined and found evidence of differences in banner visual processing for high and low median thinking viewers. High median thinking viewers exhibited sensitivity to banner complexity. Their time of global-to-local processing switch appeared to earlier for lower complexity banner ads. They also exhibited larger total scan path than low median thinkers when the complexity was low. These findings suggested that, as high median thinking viewers quickly switched from banner gist to the processing of local details for low complexity banners, the scattered distribution of information in the ad made it necessary to integrate information across spatial regions. It could be this integration effort that resulted in the large total scan path. Low median thinkers' were either late at the global-to-local switch or they were less prone to integrate information in the face of scattered information distribution in low complexity banner ads.

For banner ads of medium and high information complexity, information gained from local analysis effectively helped comprehension and the need of spatial integration was low. The high mean fixation duration for banner ads of intermediate complexity (see Table 3 and Fig.1) suggested deeper processing (and better understanding) [19] was achieved for these banners. In contrast, the mean fixation duration was the lowest for high complexity banners, suggesting fast and shallow processing of each information source. It should be noted that the medium complexity banners not only enjoyed the longer mean fixation duration, they also had the lowest number of 
fixations and the smallest total scan paths. These banners appeared to require the least amount of efforts in eye tracking or, in other words, were high in perceptual fluency. As such, they were rated to be as clear as the low complexity ones. Medium complexity ads also received deeper processing to achieve reasonable level of ad comprehension. They were similarly liked (in terms of willingness to click) as the high complexity banners. Information complexity in the banner ads served to guide viewers' eye tracking behavior.

The willingness to click on the ad was associated with small total scan path, small numbers of fixation and long mean fixation duration. Since the direction of these eye tracking measures were suggestive of low efforts, or high fluency, in eye tracking, a mediation analysis was performed to examine if fluency mediated the relationship. It was interesting to see that the analysis indeed reveal a mediating role of fluency but the mediation was significant only for high, not low, median thinkers. This and other finding about the effect of median thinking suggested that high median thinking viewers are characteristic of flexible perceptual processing that was responsive to information complexity of the banner ads. They integrate information across spatial regions when necessary. Eye tracking parameters could be used to predict their banner evaluation as they relied on the experienced fluency in eye tracking for banner evaluation. These findings on the viewing behavior of high median thinkers are consistent with the general framework of median thinking [9] in that they help support flexible, situationally appropriate manner of information processing.

A practical implication of current finding is that, for Chinese users that exhibited an overall greater extent of median thinking, banner ads are better designed to decrease the total scan path and the number of fixation as well as to increase mean fixation duration since high median thinkers tend to rely on the experienced eye tracking fluency for ad evaluation. It is worthwhile for future research to direct efforts in uncovering visual design elements that are associated with these patterns of eye tracking performance. It is also of interest to see if web page design that increased experienced processing fluency may also contribute to positive banner evaluation. As a final note at a more general level, current findings also demonstrated the potentials of eye tracking measures in predicting user's evaluation of the banner ad.

\section{References}

1. Benway, J.P.: Banner Blindness: The Irony of Attention Grabbing on the World Wide Web. Proceedings of the Human Factors and Ergonomics Society Annual Meeting 42(5), 463-467 (1998), doi:10.1177/154193129804200504

2. Burke, M., Gorman, N., Nilsen, E., Hornof, A.: Banner ads hinder visual search and are forgotten. Paper Presented at the CHI 2004 Extended Abstracts on Human Factors in Computing Systems, Vienna, Austria (2004)

3. Nielsen, J., Pernice, K.: Eyetracking web usability. New Riders Pub. (2010)

4. Dreze, X., Hussherr, F.X.: Internet advertising: Is anybody watching? Journal of Interactive Marketing 17(4), 8-23 (2003)

5. Pieters, R., Wedel, M.: Ad Gist: Ad Communication in a Single Eye Fixation. Marketing Science 31(1), 59-73 (2012), doi:10.1287/mksc.1110.0673 
6. Janiszewski, C.: The influence of display characteristics on visual exploratory search behavior. Journal of Consumer Research 25(3), 290-301 (1998)

7. Pagendarm, M., Schaumburg, H.: Why are users banner-blind? The impact of navigation style on the perception of web banners. Journal of Digital Information 2(1) (2006)

8. Hai, L., Zhao, L., Nagurney, A.: An integrated framework for the design of optimal web banners. Netnomics 11(1), 69-83 (2010)

9. Yang, C.F.: Multiplicity of Zhong Yong studies. Indigenous Psychological Research in Chinese Societies 34, 3-96 (2010)

10. Ji, L.J., Lee, A., Guo, T.: The thinking styles of Chinese people. In: Bond, M. (ed.) The Handbook of Chinese Psychology, 2nd edn., pp. 155-167. Oxford University Press (2010)

11. Lin, S.C., Wang, M.Y.: ZongYong affects the attention and memory performance for emotional stimuli. Paper Presented at the 7th Chinese Psychologist Conference, Taipei, Taiwan (2011)

12. Huang, C.L., Lin, Y.C., Chung, Y.C.: The relationship between ZhongYong thinking and the flexibility of attentioanl switch between global and local processing (2009) (Unpublished manuscript)

13. Castelhano, M.S., Mack, M.L., Henderson, J.M.: Viewing task influences eye movement control during active scene perception. Journal of Vision 9(3), 1-15 (2009)

14. Mills, M., Hollingworth, A., Van der Stigchel, S., Hoffman, L., Dodd, M.D.: Examining the influence of task set on eye movements and fixations. Journal of Vision 11(8), 1-15 (2011)

15. Shannon, C.E.: A mathematical theory of communication. . Bell System Technical Journal 27(4), 379-423, 27(4), 623-656 (1948)

16. Huang, C.-L., Lin, Y.-C., Yang, C.-F.: Revision of the Zhong Yong Belief-Value Scale. Indigenous Psychological Research in Chinese Societies 38, 3-41 (2012)

17. Kao, C.T., Wang, M.Y.: The right level of complexity in a banner ad: Roles of construal level and fluency. Paper to be Presented at the 15th International Conference on HumanComputer Interaction, Las Vegas, USA (2013)

18. Tsai, C., Thomas, M.J.: When do feelings of fluency matter? How abstract and concrete thinking influence fluency effects. Psychological Science 22(3), 348-354 (2011)

19. Cowen, L., Ball, L.J., Delin, J.: An eye-movement analysis of web-page usability. In: Proceedings of Human Computer Interaction 2002, pp. 317-335 (2002) 